\title{
Robotic Catheter Cardiac Ablation Combining Ultrasound Guidance and Force Control
}

\author{
Samuel B. Kesner and Robert D. Howe \\ Harvard School of Engineering and Applied Sciences, Cambridge, MA, USA \\ \{skesner, howe\}@seas.harvard.edu
}

\begin{abstract}
Cardiac catheters allow physicians to access the inside of the heart and perform therapeutic interventions without stopping the heart or opening the chest. However, conventional manual and actuated cardiac catheters are currently unable to precisely track and manipulate the intracardiac tissue structures because of the fast tissue motion and potential for applying damaging forces. This paper addresses these challenges by proposing and implementing a robotic catheter system that use 3D ultrasound image guidance and force control to enable constant contact with a moving target surface in order to perform interventional procedures, such as intracardiac tissue ablation. The robotic catheter system, consisting of a catheter module, ablation and force sensing end effector, drive system, and image-guidance and control system, was commanded to apply a constant force against a moving target using a position-modulated force control method. The control system uses a combination of position tracking, force feedback, and friction and backlash compensation to achieve accurate and safe catheter-tissue interactions. The catheter was able to maintain a $1 \mathrm{~N}$ force on a moving motion simulator target under ultrasound guidance with $0.08 \mathrm{~N}$ RMS error. In a simulated ablation experiment, the robotic catheter was also able to apply a consistent force on the target while maintaining ablation electrode contact with 97\% less RMS contact resistance variation than a passive mechanical equivalent. In addition, the use of force control improved catheter motion tracking by approximately 20\%. These results demonstrate that 3D ultrasound guidance and force tracking allow the robotic system to maintain improved contact with a moving tissue structure, thus allowing for more accurate and repeatable cardiac procedures.
\end{abstract}

\section{$1 \quad$ Introduction}

Advances in cardiac catheter technology allow physicians to treat a range of conditions

This work was supported by the U.S. National Institutes of Health under Grant NIH R01 HL073647 
inside the beating heart while avoiding both the invasiveness of opening the chest and the cognitive impairment risks associated with cardiopulmonary bypass (Baim 2005, 992; Murkin et al. 1999, 1498-1501; Roach et al. 1996, 1857-1864). However, the majority of catheters currently used for cardiac interventions only allow for slow manual motions of the catheter tip and are unable to control the forces applied to the tissue surfaces. Commercially available robotic catheter systems, such as the Artisan Control Catheter (Hansen Medical, Mountain View CA, USA) or the CorPath Vascular Robotic System (Corindus Vascular Robotics, Natick MA, USA), achieve manual catheter manipulation speeds while allowing the operator to utilize robotic teleoperation to reduce radiation exposure (Camarillo et al. 2008, 1262-1273; Beyar 2010, 207-213; Jayender, Azizian, and Patel 2008, 858-871). However, neither the manual nor the commercial robotic catheter systems are able to compensate for the fast cardiac motion or regulate the forces applied to the tissue surface.

The goal of our work is to enable a robotic catheter to track the fast motions of the heart while controlling the forces applied by the catheter end effector to the tissue in order to improve the safety and efficacy of medical procedures. This objective is achieved through the use of 3D ultrasound (3DUS) guidance, active motion compensation, and catheter tip force control. One medical application that may benefit from this technology is the radiofrequency (RF) ablation of cardiac tissue. Ablation is used by interventional cardiologists and cardiac surgeons to destroy cardiac conduction pathways that contribute to arrhythmias, or heart beat abnormalities (Huang, Huang, and Wilber 2000). The outcome success of this procedure is dependent on the electrode contact with the tissue and force application, and therefore can benefit from the robotic system proposed here (Okumura, Johnson, and Packer 2007, S318; Shah et al. 2010, 1038-1043; Shah et al. 2011, 277; Kalman et al. 1997, 8-18). In addition to cardiac ablation, other applications of this robotic system include beating heart cardiac valve repair and replacement and the delivery of implants to moving structures inside of the beating heart, such as septal defect repair implants.

In previous work, we have demonstrated in vivo the ability of the robotic catheter system to compensate for the fast motion of the heart (Kesner and Howe 2011c, 1-11). In addition, a custom catheter tip force sensor was developed to enable the catheter to maintain a constant force against a target (Kesner and Howe 2012). However, the catheter force control system has to date only been evaluated on the benchtop using noise-free simulated position signals rather than noisy ultrasound image-derived signals (Kesner and Howe 2011a, 1-5; Kesner and Howe 2011b, 
1589-1594). Other work in cardiac motion compensation has focused primarily on interacting with the exterior of the beating heart (Ginhoux et al. 2005, 67-79; Bebek and Cavusoglu 2007, 468480; Nakamura, Kishi, and Kawakami 2001, 2014-2019; Richa, Bó, and Poignet 2010, 4579-4584). In addition, the previous work in robotic catheters has primarily focused on teleoperation and position control (Camarillo et al. 2008, 1262-1273; Beyar 2010, 207-213; Jayender, Azizian, and Patel 2008, 858-871). To the authors' knowledge, the work presented here represents the first time 3DUS image guidance and force control has been used to enable a robotic catheter to accurately interact with a moving target. As shown below, the combination of force feedback and 3DUS servoing improves both the force and position control performance. Furthermore, this paper presents the first steps towards applying the 3DUS-guided and motion compensated catheter system to a clinical procedure, improving cardiac ablation using force control.

The following section presents the robotic catheter system, the force sensing and ablation end effector, and the force control method. Next, the paper presents the evaluation of the system, including benchtop experiments examining the control method, water tank evaluations of the image guidance force controller, and experiments evaluating the efficacy of the robotic catheter system during a simulated cardiac ablation as compared against a passive manual catheter. Finally, this paper concludes with a discussion of the implications and limitations of the results and future applications of the technology. This work demonstrates the potential benefits of integrating motion-compensation and force control with cardiac intervention catheters.

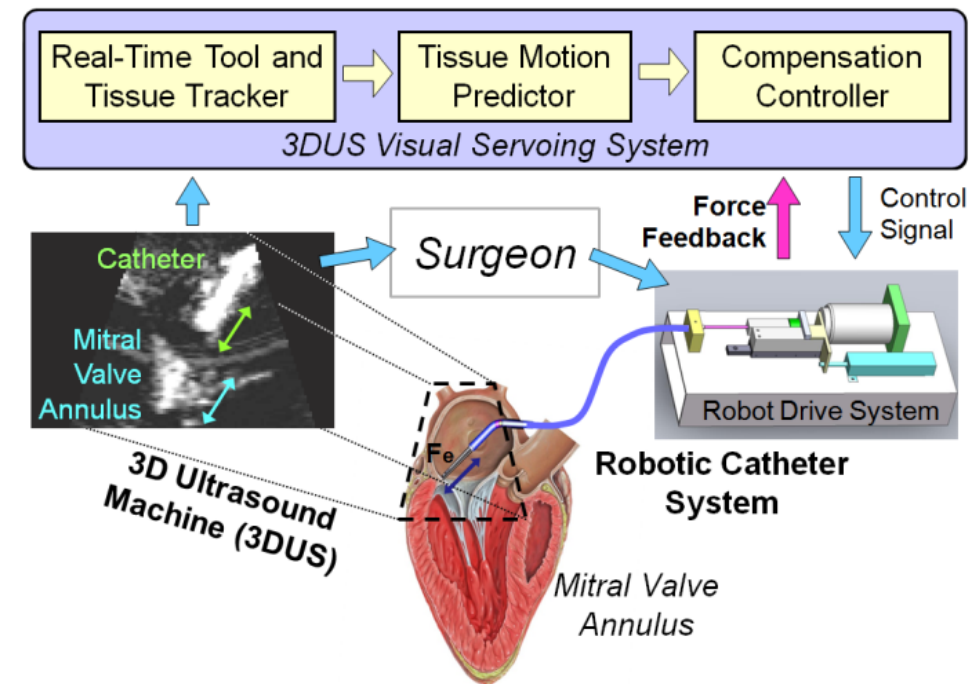

Figure 1: The robotic system servos the catheter using 3DUS guidance and force feedback. 
The goal of the robotic catheter system is to use real-time 3DUS to measure the target tissue motion and then drive the actuated catheter to synchronize with the motion and while interacting with and repairing a cardiac target tissue. The catheter system is designed to rapidly servo along one degree of freedom at speeds and displacements sufficient for tracking certain cardiac structures including the human mitral valve annulus (Kesner and Howe 2011c, 1-11; Kettler et al. 2007, 1290-1295). The system (Figure 1) is composed of three main modules: the drive system that actuates the catheter, the catheter module that is inserted through the vasculature into the heart, and the 3D ultrasound visual servoing system that tracks the tissue and commands the catheter to follow the motion.

The catheter drive system (Figure 3.1) is composed of a linear voice coil actuator (NCC20-18-02-1X, H2W Technologies Inc, Valencia CA; $50.8 \mathrm{~mm}$ travel, $26.7 \mathrm{~N}$ peak force), a linear ball bearing slide (BX3-3, Tusk Direct, Inc., Bethel CT), and a linear potentiometer position sensor (LP-50F, Midori America Corp, Fullerton CA, linearity: $\pm 0.5 \%$ ) that are able to rapidly adjust the catheter position. The catheter module consists of a sheath, a guidewire, and the end effectors required for each specific repair procedure. The sheath is an $85 \mathrm{~cm}$ long section of flexible Teflon or Nylon tubing that encloses the guidewire, a close-wound stainless steel spring that is easily bent but can apply significant compressive forces without buckling. During a

procedure, the sheath is inserted from a peripheral blood vessel into the heart and then fixed in place while the drive system servos the guidewire inside the sheath to compensate for the heart motion and regulate the forces applied to the cardiac tissue.

\subsection{D Ultrasound Servoing System}

The visual servoing system utilizes a 3DUS machine (Figure 1, SONOS 7500 with X4 Ultrasound Transducer, Philips Healthcare, Andover, MA, USA) that streams volumes at $28 \mathrm{~Hz}$ and a guidance system to track and predict the tissue motion in order to determine the real-time position of the cardiac tissue and control the catheter (Novotny et al. 2007a, 458-464; Novotny et al. 2007b, 2655-2660; Yuen, Novotny, and Howe 2008, 3875-3880; Yuen et al. 2009, 1355). 
The ultrasound servoing system (Figure 3) streams 3D image volumes from the ultrasound scanner to an image processing computer via Ethernet. A GPU-based Radon transform algorithm finds the catheter axis in real-time. The target tissue is then located using the flashlight tissue tracker algorithm, which projects the instrument axis forward through the image volume until tissue is encountered (Yuen et al. 2010). This method allows the clinician to designate the target to be tracked by simply pointing at it with the catheter. To compensate for the 50-100 ms delay in image acquisition and processing, an extended Kalman filter (EKF) estimates the current tissue location based on a Fourier decomposition of the cardiac cycle (Yuen et al. 2009, 1355). Previous in vivo experiments using this servoing system showed that a rigid instrument system was capable of accurate tracking within the heart, with an RMS error of 1.0 mm. See (Yuen et al. 2009, 1355; Yuen, Novotny, and Howe 2008, 3875-3880; Novotny et al. 2007b, 2655-2660; Novotny et al. 2007a, 458-464; Kesner, Yuen, and Howe 2010, 168-178) for a detailed description of the 3DUS visual servoing system.

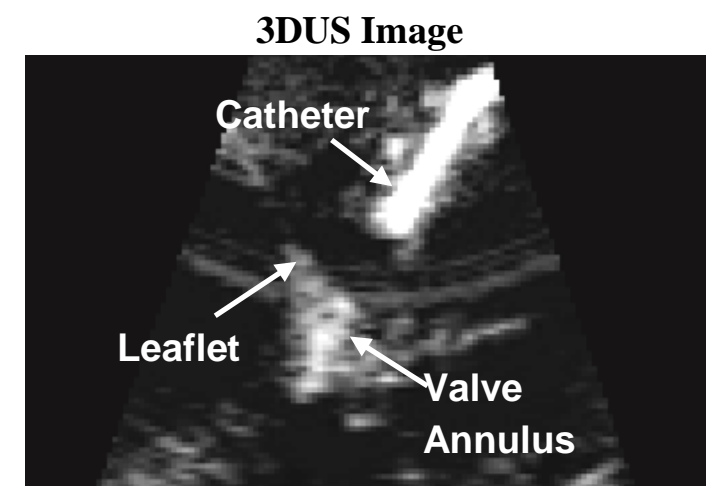

Figure 2: Ultrasound image showing the catheter, mitral valve annulus, and mitral valve leaflets. 


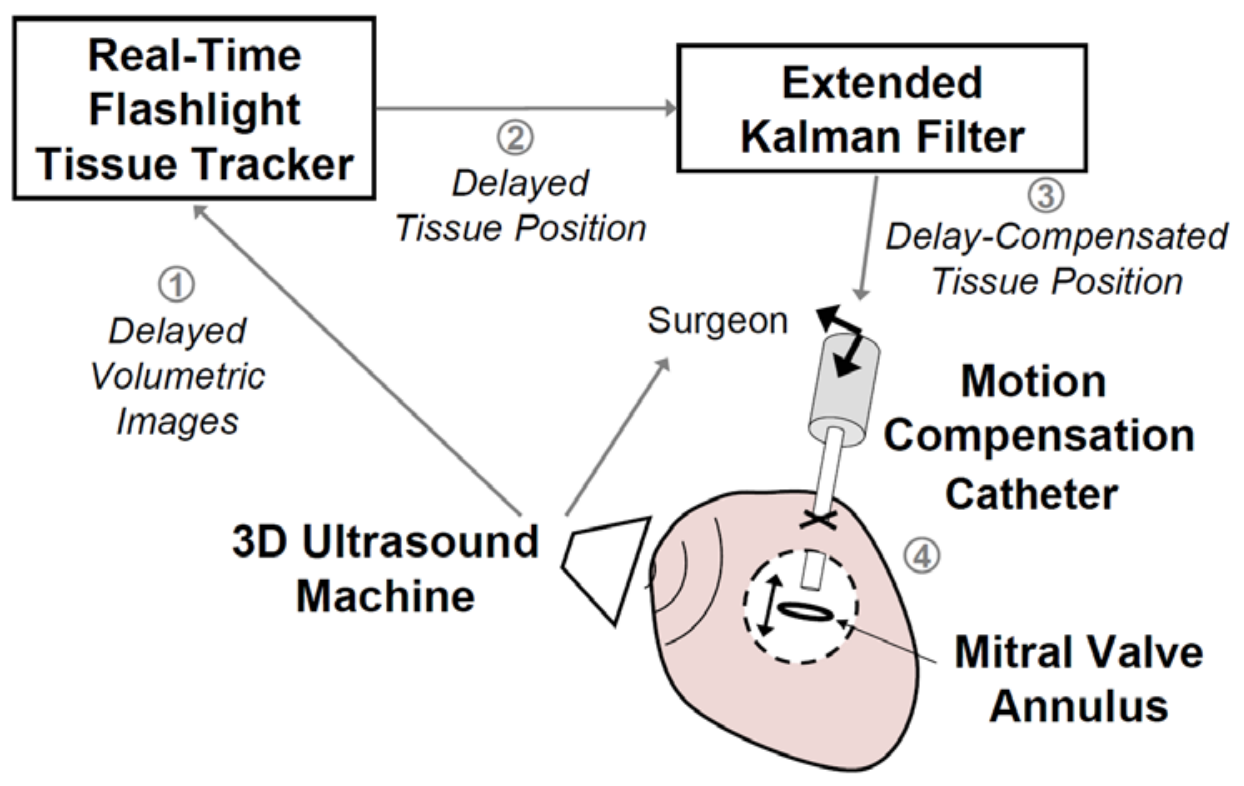

Figure 3: The 3DUS servoing system uses 3D ultrasound imaging to automatically synchronize the motion of an actuated device with a tissue target. The circled numbers indicate the order of data flow through the system: 1) The time-delayed 3DUS images, 2) the tissue positions generated by the tissue tracking algorithm, 3) the delaycompensated tissue positions, and 4) the resultant tracking position of the motion compensation catheter.

\subsection{Force Sensor}

In addition to the robotic catheter, a novel integrated force sensing and ablation end effector is presented here for the first time (Figure 4). The design goal of the ablation tool is to enable the catheter system to apply RF energy to the fast-moving tissue inside the heart while applying a constant normal force. The functional requirements of the ablation end effector are to sense forces, to ablate tissue using a clinical RF generator with a similar efficacy to conventional ablation catheters, and to be robust enough to operate in the intracardiac environment. The device consists of a force sensor described in (Kesner and Howe 2011a, 1-5), a stainless steel electrode, and a fine wire that runs through the catheter body to connect the electrode to the external RF current generator. The current prototype is approximately $5 \mathrm{~mm}$ in diameter and is created using rapid prototyping 3D printing technology. The size of the end effector can be further reduced with improved fabrication such as laser micromachining and metal laser sintering. Figure 4b presents examples of the RF ablation lesions created with this tool on porcine skeletal muscle tissue (RF generator: Stockert 70, Biosense Webster, Diamond Bar, California, USA). Figure 5 presents a schematic diagram of the force sensor component of the 
ablation end effector and example force sensor performance data. The force sensor converts applied forces into small deflections of a reflective surface supported by flexures. The reflective surface modulates the amount of light reflected back to a fiber optic sensor, which is calibrated and used to determine the amount of applied force. NiTi wires flexures ( $0.25 \mathrm{~mm}$ diameter) are arranged in a perpendicular configuration, which allows for large defections along the axis of the catheter but resists lateral deflections, has little hysteresis, and prevents rubbing of the sensor components. Superelastic NiTi flexures were selected for overload protection. A fiber optic transducer was selected for this sensor because it is inexpensive, easy to implement, and requires no electrical components within the catheter (Webster 1988). Integrating the transducer into the sensor is as simple as inserting the fibers and fixing them in place with adhesive.

The Objet Connex500 3D printer (Objet Geometries Ltd, Billerica, MA, USA) was used to fabricate the catheter ablation and force sensor end effector. This printer has a minimum resolution of 16 microns and can print with a range of photopolymers, from a stiff acrylic plastic to a rubber-like, flexible plastic (see (Objet Geometries Ltd. 2010)). For this work, the Verowhite photopolymer was selected because it is a stiff plastic (2 GPa) with a high tensile strength (50 $\mathrm{MPa})$.

The catheter tip sensor was calibrated and tested with a commercial 6-axis force torque sensor (Mini40, ATI Industrial Automation, Apex, NC, USA). The 3D printed sensor was manually loaded against the ATI sensor with a varying force profile. The signal from the fiber optic sensor was amplified with a digital fiber amplifier (E3X-DA21-N, Omron Electronics LLC Industrial Automation, Schaumburg, IL, USA) before digital acquisition (DAQCard-6024E, National Instruments Corp., Austin TX, USA). See (Kesner and Howe 2011a, 1-5) for a more detail description of the 3D printed force sensor technology.
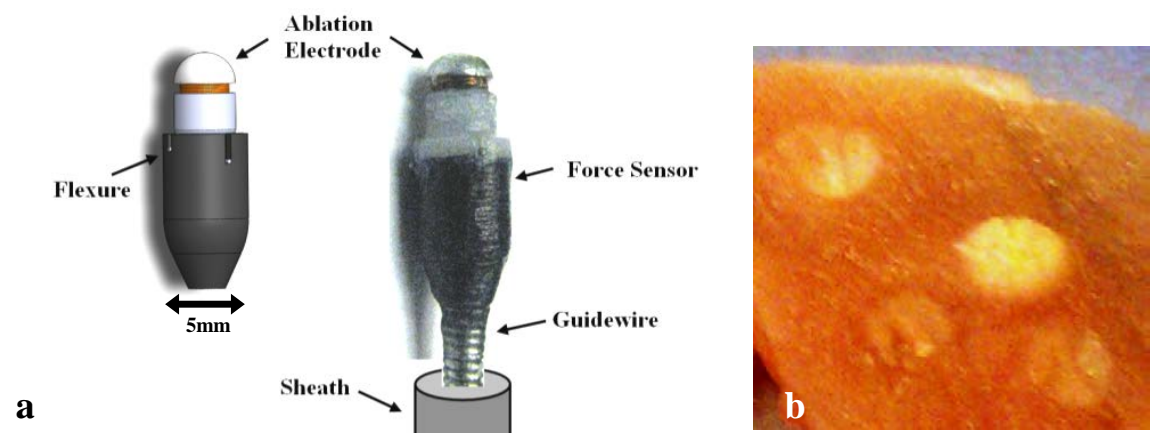

Figure 4: a) Ablation end effector solid model and prototype. b) Tissue sample (porcine skeletal muscle) ablated with the RF ablation end effector. Lesions are approximately $4 \mathrm{~mm}$ in diameter. 

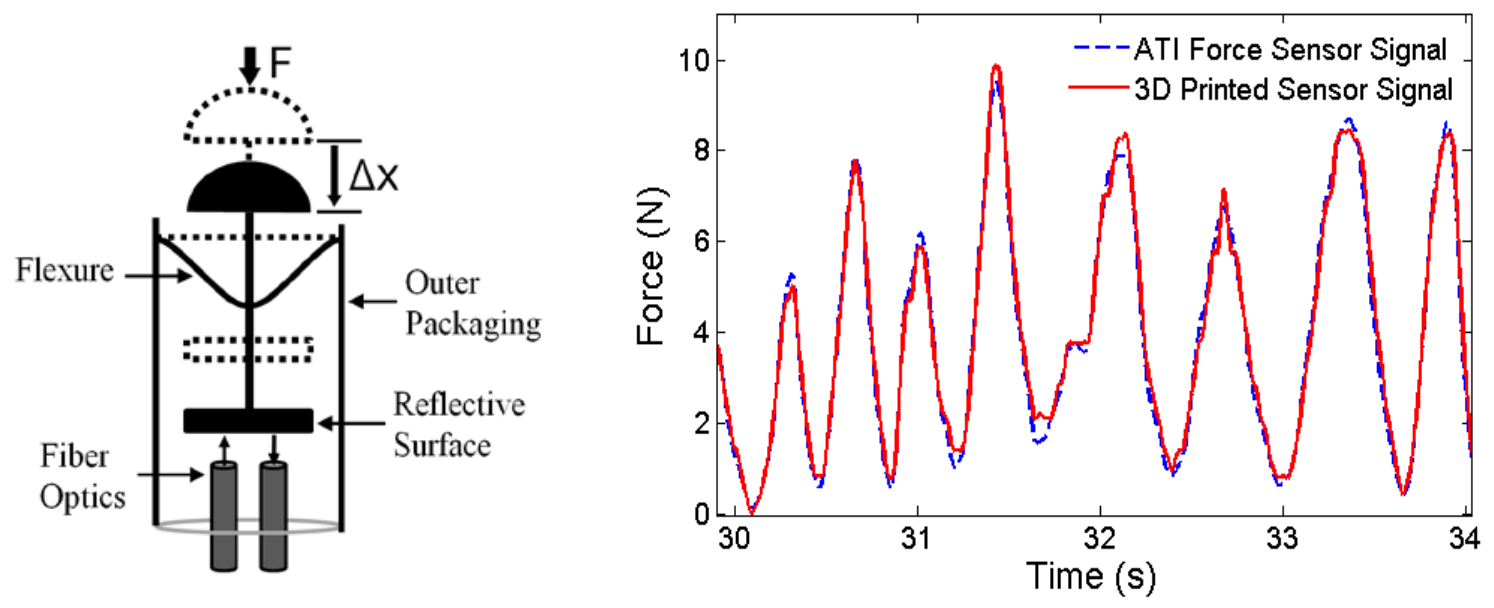

Figure 5: Left: Catheter tip force sensor schematic. Right: 3D printed force sensor response to time-varying loading

(Kesner and Howe 2011a, 1-5)

\subsection{Force Control Method}

The objective of the control system is to apply a desired force on a fast moving target with the robotic catheter end effector. A standard error-based force control approach will not work for the robotic catheter system because of the limitations identified in (Kesner and Howe 2011c, 1-11), including backlash and friction in the catheter transmission system (Eppinger and Seering 1987, 904-909; Townsend and Salisbury Jr 1987, 883-889). To overcome these issues, we propose a method that uses the force error term to modulate the commanded position trajectory of the catheter. This approach is similar to the inner position loop force control approaches used to implement force control on high-friction industrial manipulators (Maples and Becker 1986, 695702) and methods presented by Villani et al in (Chiaverini, Siciliano, and Villani 1999, 273-285). This approach results in improved system stability and allows for the controller to directly compensate for the catheter friction and backlash in the inner position loop as these limitations are position and velocity dependent (Kesner and Howe 2011c, 1-11). See Figure 6 for a block diagram of the control system.

In this force control approach, the drive system is commanded to follow a desired position, $x_{d}$, that is the sum of the position of the moving target, $x_{e}$ and the position offset required to maintain the desired force, $x_{f}$

$$
x_{d}=x_{e}+x_{f}
$$


The force modulation term is

$$
x_{f}=K_{f}\left(F_{d}-F_{e}\right)+K_{f i} \int\left(F_{d}-F_{e}\right) d t
$$

where $F_{d}$ is desired force, $F_{e}$ is the force applied to the environment, and $K_{f}$ and $K_{f i}$ are controller gains. The drive system is commanded to follow the desired position trajectory with a standard PID controller running at $1 \mathrm{kHz}$.

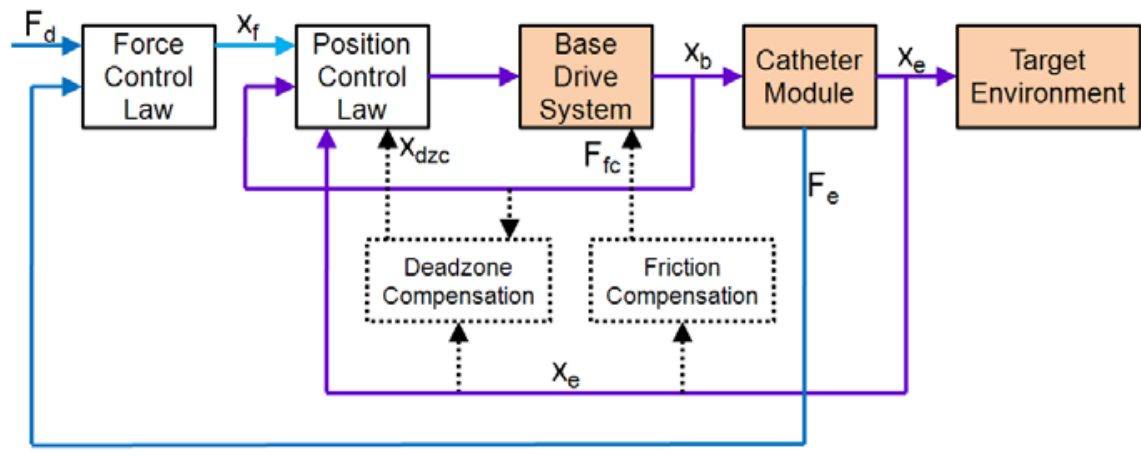

Figure 6: The force control system block diagram. The control system uses force and position feedback as well as compensation terms to command the catheter to maintain a desired force on a moving target.

\subsection{Compensation Methods}

Compensation terms are introduced to the position feedback loop to improve the control system performance, indicated in the block diagram in Figure 6 by dotted lines (Kesner and Howe 2011c, 1-11). Friction compensation assumes a Coulombic friction model for the catheter and then feeds forward the friction force $F_{f c}$, based on an observer that predicts the velocity (Kesner and Howe 2011c, 1-11). The friction force is determined during operation through an estimation routine that utilizes the motor current values and is dominated by the catheter system design (materials, geometry) and sheath configuration bend angle $(\theta)$. The bend angle $(\theta)$ can be determined during a procedure using a number of methods, including bend sensors embedded along the length of the catheter sheath (Prisco ), electromagnetic tracking (Wilson et al. 2008, 69182B-1), and the extraction of catheter bend angle information from medical images (most likely X-ray fluoroscopy) during the procedure. Backlash compensation adds an additional term 
to $x_{d}$ that adjusts the desired base position to overcome the deadzone behavior in the catheter module. The amount of compensation, $x_{d z c}$, is determined using a catheter-specific deadzone model presented in (Kesner and Howe 2011c, 1-11):

$$
x_{d z c}=\theta\left(D_{s h}-D_{g w}\right)
$$

where $D_{s h}$ is the inner diameter of the sheath and $D_{g w}$ is the diameter of the guidewire. The compensation term $x_{d z c}$ is either added or subtracted from $x_{d}$ based on the direction of target motion and the position of the guidewire relative to the deadzone region. While the deadzone value is sensitive to the bend angle, this parameter can be accurately measured with the methods described above and will not vary greatly over the course of a procedure as the sheath is constrained by the vasculature between the entry site and the heart.

\subsection{Force Controller Limitations}

The performance of the force control method described here is limited by a number of factors, including the accuracy of the tissue motion tracking, the compensation terms, and the unmodeled effects of the environment.

The most significant limitation of the controller is its dependence on accurate target motion information for motion compensation and force regulation. The controller relies on the periodic motion of the cardiac tissue to enable accurate predictive position estimations (Yuen, Novotny, and Howe 2008, 3875-3880; Kesner and Howe 2011c, 1-11). If the tissue motion deviates greatly from the previous periodic trajectory, the desired position $\left(x_{d}\right)$ component of the position control loop will be incorrect because the estimated position of the environment $\left(x_{e}\right)$ will not match the real tissue motion. Possible sources of target motion tracking errors include external disturbances like arrhythmias or ectopic beats, unmodeled motions like respirations, changes in the tissue motion due to the forces applied by the catheter, and failures of the motion prediction algorithm to converge on the correct trajectory due to poor imaging quality or tool positioning (Yuen et al. 2008; Yuen et al. 2009, 1355; Kesner and Howe 2011c, 1-11).

Another limitation of the force controller arises from changes to the physical catheter system. The controller assumes a static model for the system performance parameters for a given catheter configuration. For example, the friction forces and backlash parameters are fixed 
for each catheter position and orientation. While assuming a static catheter configuration is reasonable while operating in the constrained workspace inside the heart, other factors, such as blood clots developing between the catheter guidewire and sheath, can change the friction and backlash model parameters. To overcome these model limitations, the backlash and friction are determined once the catheter is in position but before it has engaged the tissue to include any effects from recent changes in the system or configuration.

In certain situations, the model-predicted deadzone width must be increased to account for the deformation of the sheath and guidewire caused by significant catheter friction (Kesner and Howe 2011c, 1-11). In this study, $x_{d z c}$ was doubled for certain trials to account for the increased deadzone width caused by significant friction values as high as $2 \mathrm{~N}$. This frictiondependant backlash behavior has been reported in previous work (Kesner and Howe 2010, 10591065; Kesner and Howe 2011c, 1-11).

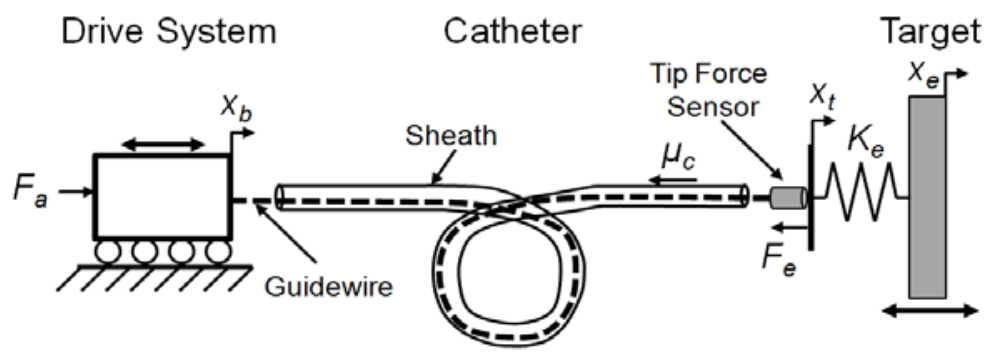

Figure 7: Benchtop evaluation experimental setup

\section{Benchtop Experimental Evaluation}

The force control methods proposed above were first evaluated on the benchtop to characterize the system and determine how well the catheter can maintain a desired force against a fast moving target. Based on our previous studies of fast motion compensating with a catheter, the important experimental variables to examine are the catheter bend angle $(\theta)$ and the speed and trajectory of the target (Kesner and Howe 2011c, 1-11). See Figure 7 for a diagram of the benchtop experimental setup used to evaluate the controller designs. Ground truth values were determined using a number of appropriate sensing modalities, including electromagnetic trackers 
(3D Guidance trakSTAR system, Ascension Technology Corporation, Milton VT, USA) to measure the catheter tip trajectory, a linear potentiometer position sensor integrated into the motion simulator mechanism to sense the target position, and a commercial force sensor (LCFD1KG, Omega Engineering, Stamford, CT, USA; range: $10 \mathrm{~N}$, accuracy: +/-0.015 N) and the catheter force sensor to determine the applied force values.

The first set of experiments examined the performance of the force control schemes while interacting with a target following a $12 \mathrm{~mm}$ peak-to-peak, $1 \mathrm{~Hz}$ sinusoidal trajectory in three sheath bend configurations: $0^{\circ}, 180^{\circ}$, and $360^{\circ}$. The friction, modeled as simple Coulombic friction, increases approximately linearly with bend angle (Kesner and Howe 2010, 1059-1065). The width of the backlash deadzone, described in eqn. (3), is also a function of the bend angle and can be accurately predicted with the deadzone width model first presented in (Kesner and Howe 2010, 1059-1065).

A sinusoidal trajectory was selected as an initial experimental evaluation trajectory because it is the dominant component of cardiac motion, as demonstrated by the spectral decomposition in (Kettler et al. 2007, 1290-1295). For a more realistic experiments, a human mitral valve annulus trajectory was also used below to evaluate the system (Kettler et al. 2007, 1290-1295).

To evaluate the performance of the proposed force control methods, the system was tested with three controller configurations: 1) the force-modulated position controller in eqn. (2), 2) the controller with an added friction compensation term, and 3) the controller with both friction and deadzone compensation terms. The force-modulation gains, $K_{f}$ and $K_{f i}$, were tuned for best stable performance and kept constant for all of the experiments.

\subsection{Force control methods comparison}

Figure 8 presents a comparison of the controller performance applying a constant force ( $1 \mathrm{~N}$ ) against the motion simulator target with the catheter in a $360^{\circ}$ bend configuration. The target was covered with a compliant foam with a stiffness $\left(K_{e}\right)$ of approximately $0.25 \mathrm{~N} / \mathrm{mm}$ to simulate cardiac tissue.

The results in Figure 8 demonstrate that both friction and deadzone compensation greatly improve the force tracking. Significant tracking errors can be seen when the target changes direction in both Figure 8b and Figure 8c. These errors are because the controllers in these plots 
do not compensate for the deadzone region behavior. Experimentally, this behavior appears as if the tip of the catheter is delayed in responding to the changes in the target trajectory. Deadzone compensation, demonstrated in Figure 8d, significantly improves the tracking by adjusting the desired position to remove the backlash effects of the deadzone. Friction compensation also improves tracking by cancelling the friction resistance in the sheath, as seen in the improvement in performance between Figure 8b and Figure 8c.

Figure 9 summarizes the performance results of the three force controllers for each of the three catheter configurations. The average performance of each the controllers, presented in Figure 9a as the RMS deviation from the desired force, shows that the compensation terms significantly improve the catheter system's force tracking ability. For example, the RMS error for the $360^{\circ}$ bend configuration decreases by over $45 \%$ when friction compensated was added and by almost $86 \%$ when both friction and deadzone compensated were added.

The maximum deviations from the desired force are expressed as the peak-to-peak value, the difference between the maximum and minimum tip force value during each experiment. These deviations are often greatest during the changes in the target's direction of motion (Figure 8). This data, presented in Figure 9b, clearly indicates that the compensation methods reduce the deviations from the desired force. For example, friction and backlash compensation decreased the peak-to-peak variations in the $360^{\circ}$ bend configuration by almost $60 \%$. It should be noted that for the $0^{\circ}$ catheter bend configuration, the deadzone compensation does not alter the RMS or peak-to-peak values because the catheter system has no deadzone according to the backlash model in eqn. (3).

The effect of the frequency of the sinusoidal target was also investigated in this study. The target frequency was varied from $0.1-1.6 \mathrm{~Hz}$, approximately the range of possible heart rates encountered during clinical procedures (6-96 BPM). The catheter was constrained in a $180^{\circ}$ bend configuration and the control system was commanded to maintain a desired force of $1 \mathrm{~N}$ with and without friction and deadzone compensation. 

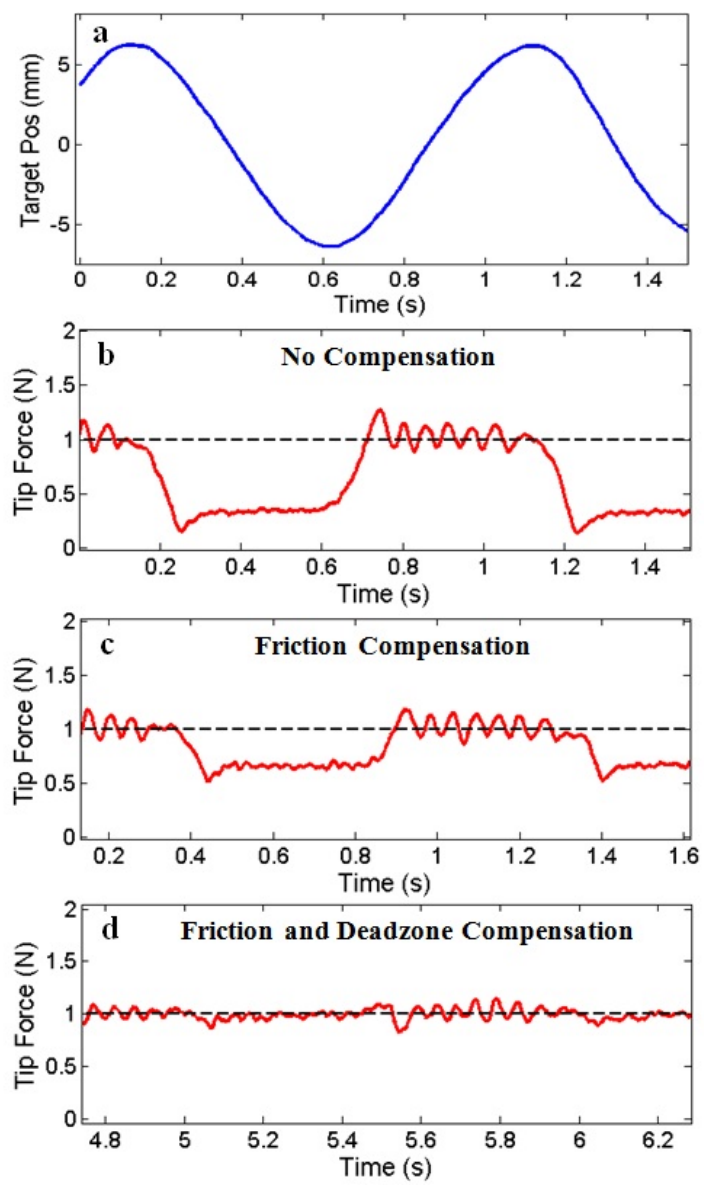

Figure 8: Sinusoid trajectory comparison. (a) $1 \mathrm{~Hz}$ sinusoidal target trajectory and (b) the catheter tip force with only force-modulated position control, (c) with the addition of friction compensation, and (d) with the addition of both friction and deadzone compensation. The bend angle is $360^{\circ}$.

The results of these experiments are summarized in Figure 10. The RMS error for both controllers was approximately constant across the frequency range, with the compensated controller performing roughly $75 \%$ better than the uncompensated controller for all of the frequencies. The peak-to-peak error increased as a function of the frequency. This trend is because, as the frequency increases, the speed at which the catheter must travel through the deadzone to maintain the desired force also increases. 

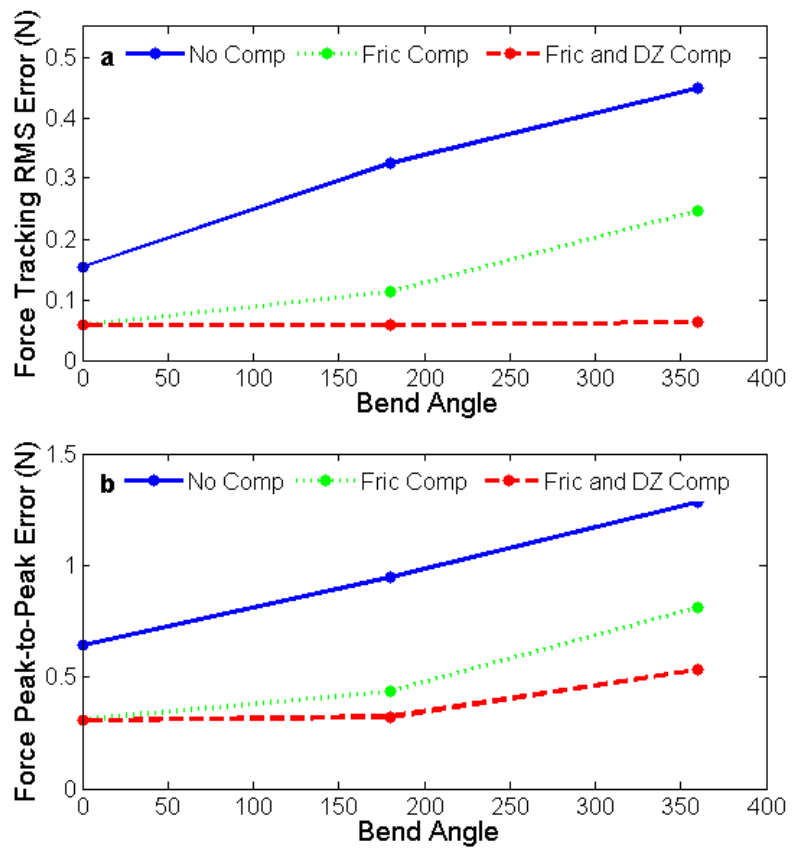

Figure 9: (a) Force tracking RMS error and (b) force tracking peak-to-peak error against a $1 \mathrm{~Hz}$ sinusoidal target as a function of bend angle for the three force control methods
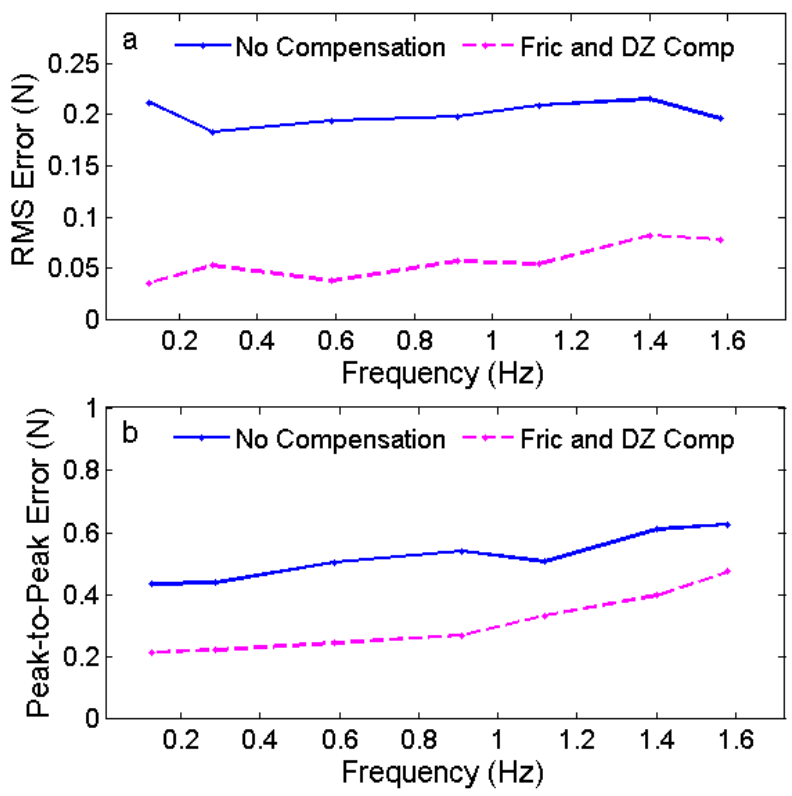

Figure 10: (a) Force tracking RMS error and (b) peak-to-peak error while tracking a sinusoidal trajectory as a function of the target frequency. 


\section{3D Ultrasound-Guided Performance Evaluation}

The force controlled catheter system was also evaluated in water tank studies under 3DUS guidance. A water tank is required to evaluate the visual servoing system because clinical ultrasound machines cannot operate in air.
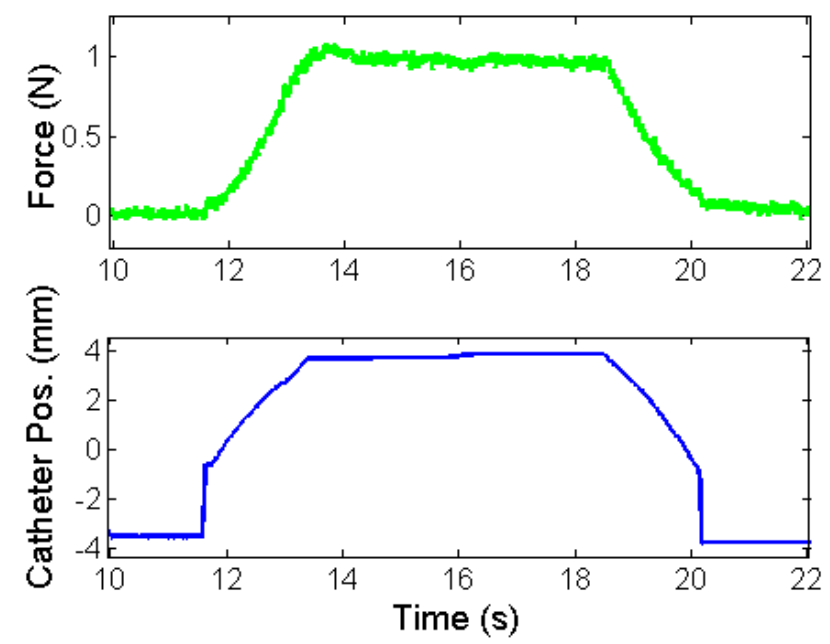

Figure 11: Engaging the target: The catheter engages and retracts from a static target using a trapezoidal trajectory to achieve the desired force.

\subsection{Engaging the Target}

One of the challenges of regulating the forces applied by the catheter tip to a target is the transition from operating in free space to applying the desired force on the fast-moving target. The process of engaging a surface is challenging because of the potential for the catheter tip to apply large and destabilizing interaction forces. Furthermore, the catheter must be able to safely retract from the target surface after the experiment is completed. To ensure that the catheter contacts the target in a controlled manner, a trapezoidal position trajectory is commanded to dictate the processes by which the tool engages and retracts from the target.

It is assumed that the catheter must maintain a constant force against the tissue in order to apply a surgical technique such as ablate, resect, or staple the tissue. In the method used here, the catheter approaches the surface at a rate of $2.5-5 \mathrm{~mm} / \mathrm{s}$, applies the desired force for $5 \mathrm{~s}$, and then retracts from the tissue at $5 \mathrm{~mm} / \mathrm{s}$. Figure 12 demonstrates engaging a static target. An alternative engagement process is for the user to manually adjust the catheter position until it 
makes first contact with the moving tissue and then switch to a force control method to maintain a constant interaction force.

The process of engaging a target is further complicated when the target is quickly moving, such as the mitral valve annulus (Kettler et al. 2007, 1290-1295). If the approach process does not consider the motion of the target and progresses at a constant velocity, the catheter tip may collide with the target surface and result in a large spike of force and possibly system instability. To prevent this issue, the moving target is virtually stabilized relative to the catheter tip by utilizing motion compensation during the approach procedure.
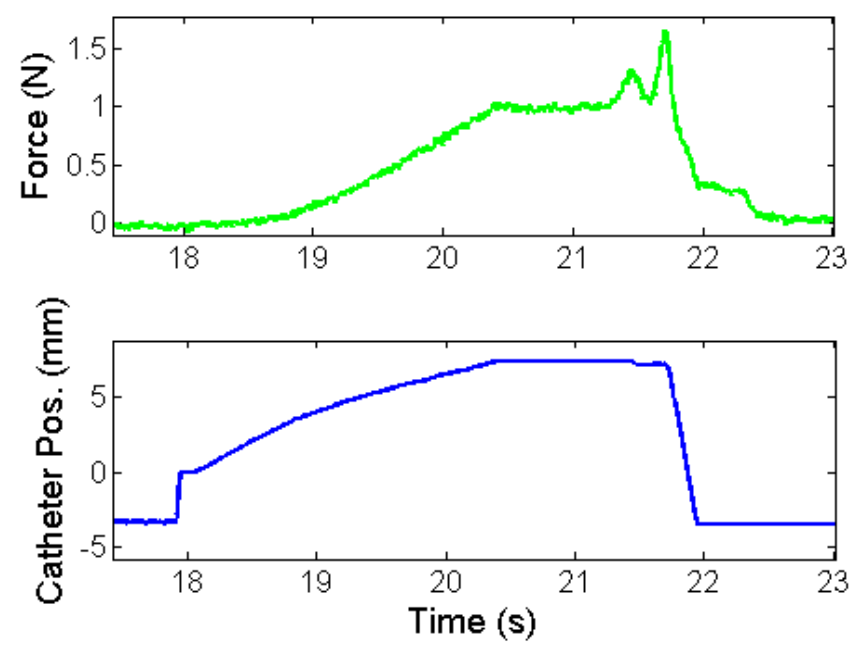

Figure 13: The catheter tip immediately pulls back into a safe position if the force threshold (1.5 N) is exceeded to prevent tissue damage.

To prevent damage to the tissue or tool caused by instability or unexpected forces applied to the catheter tip, the controller is instructed to pull back the catheter and enter a "safe mode" if a larger than expected force is sensed at the tip. As demonstrated in Figure 13, the catheter withdraws at a speed of $50 \mathrm{~mm} / \mathrm{s}$ when a force spike is recorded at the tip. This force spike, created by quickly hitting the tip of the catheter force sensor, simulates an ectopic heart beat not anticipated by the motion compensation controller, causing the catheter tip to collide with the heart wall. The force threshold, in this case $1.5 \mathrm{~N}$, determines if the controller needs to pull back the catheter into the safe mode.

In previous research we recorded forces required for interventional cardiac procedures up to $1.5 \mathrm{~N}$ (Wagner et al. 2006, 43-48). We therefore selected $1 \mathrm{~N}$ as the target force level, with the $1.5 \mathrm{~N}$ level as the upper bound. Based on the literature, this target force is likely greater than the 
optimal contact force for cardiac ablation (Shah et al. 2010, 1038-1043). The $1.5 \mathrm{~N}$ force threshold was also selected during preliminary experiments to indicate that the force tracking had diverged and to prevent any damage to the force sensor or catheter mechanisms.

Other possible metrics for determining this error state in addition to a force threshold include a catheter velocity threshold to anticipate instability or limit cycles and a system power function that includes both catheter tip velocity and force. In addition, electrocardiogram (ECG) monitoring can be used to anticipate ectopic heart beats or arrhythmias and withdraw the catheter to a safe position.

\subsection{Evaluation Results}

The catheter system was evaluated using the 3DUS tracking system in a water tank interacting with both the sinusoidal and mitral valve annulus motion target. The use of the 3DUS complicates the force tracking process because of the importance of the target position prediction, as discussed above in section 2.1. Even in the controlled environment of the water tank experiment, the system performance varied between each trial due to the quality of the EKF tissue position prediction component of the visual servoing system described in Section 2.1 (Yuen et al. 2010). In spite of these limitations, performance values of $0.08 \mathrm{~N}$ RMS errors were achieved for the sinusoidal trajectory (Figure 14 ). Without friction and deadzone compensation, force tracking results of only 0.15 N RMS were achieved relative to the sinusoidal target (Figure 14 ). Force control performance values of 0.17 N RMS errors were achieved while interacting with a mitral valve annulus motion simulator with friction and deadzone compensation (Figure 15). The target trajectory for this experiment was generated from human mitral valve annulus data (Kettler et al. 2007, 1290-1295). See Figure 14 and Figure 15 for plots of the system performance in these experiments. 

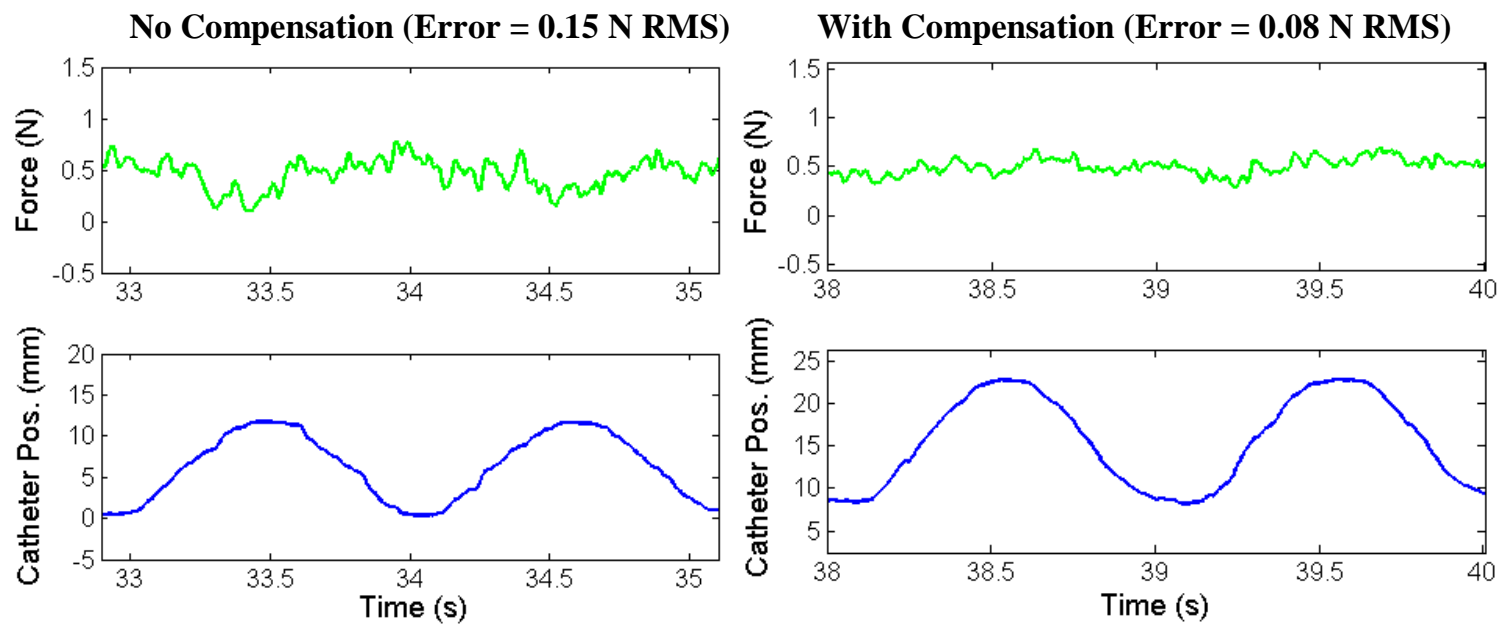

Figure 14: The force tracking results in a water tank under 3DUS guidance for the sinusoidal target trajectory. Left: Without deadzone or friction compensation $($ RMS error $=0.15 \mathrm{~N})$. Right: With compensation terms $($ RMS error $=$ $0.08 \mathrm{~N})$.
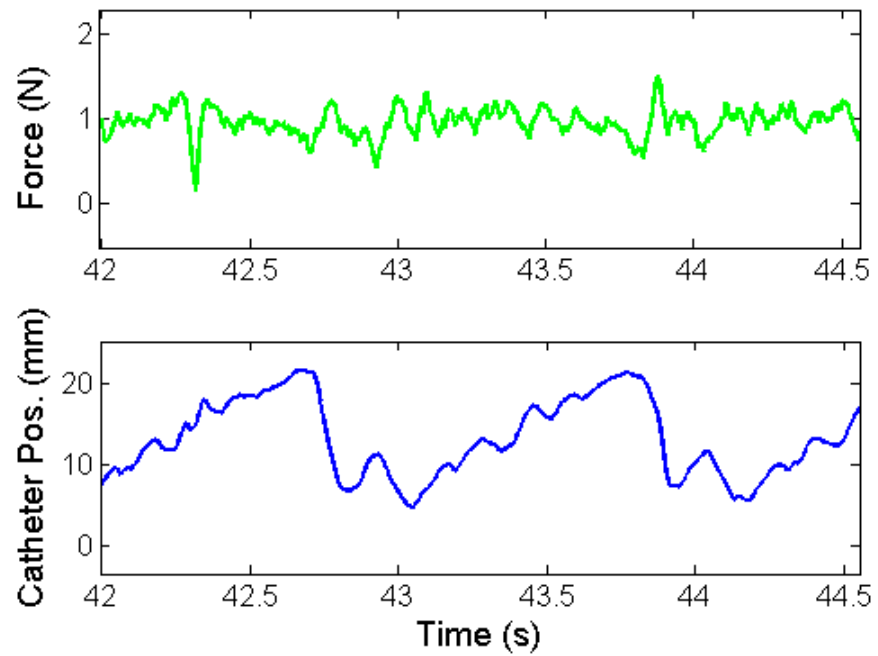

Figure 15: The force tracking results in a water tank under 3DUS guidance with a mitral valve annulus trajectory target with the compensation terms. Force tracking error $=0.17 \mathrm{~N}$ RMS.

\subsection{Motion tracking improvements due to force feedback}

In addition to providing force tracking, the catheter control system also improves the position tracking accuracy of the catheter tip. The position trajectory commanded by the 3DUS tracking algorithm follows the true position of the human mitral valve motion simulator with $1.60 \mathrm{~mm}$ RMS error. However with the addition of the force feedback controller, the catheter position is accurate to $1.31 \mathrm{~mm}$, an improvement of almost 20\%. Figure 16 illustrates this improvement. The catheter trajectory tracks the minima and maxima of the target motion better with the addition of the force feedback modulation controller. 
There are two possible explanations for this improved result. First, the backlash compensation method commands the catheter to overshoot the target position at the extremes of the motion trajectory. This behavior would provide improved motion tracking if the 3DUS guidance method was not able to track the extremes of the target motion. The more likely explanation is that the force feedback pushed and pulled the catheter into the correct positions. As the catheter tip maintains contact with the target, any deviations of the position tracking will result in an increase or decrease of the forces experienced at the catheter tip. The force feedback loop responds to this force change by modulating the catheter tip position and adjusting the catheter trajectory to better match the target. Thus, the force feedback modulation corrects some of the errors of the 3DUS tracking by correcting its position to maintain the constant force on the moving target.

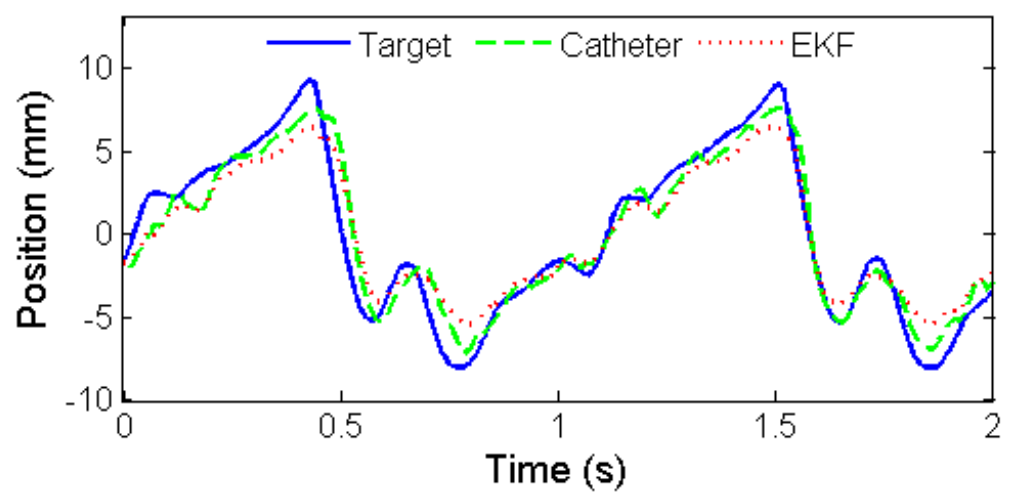

Figure 16: The position-modulation term of the force controller improves the tip trajectory tracking. The three lines are the actual target position, the desired catheter position determined by the force control system, and the EKF-predicted target position from the 3DUS-guidance system.

\section{$5 \quad$ Ablation Experiments}

The final set of experiments presented here evaluates the robotic catheter system's ability to perform a simulated interventional task, maintaining ablation electrode contact against a moving surface while applying a constant force. A number of studies have demonstrated that cardiac ablation efficacy is directly related to the forces applied by the catheter tip and the quality of the electrode-tissue contact (Okumura, Johnson, and Packer 2007, S318; Shah et al. 2010, 1038-1043; Shah et al. 2011, 277; Kalman et al. 1997, 8-18). Manually operated catheters do not adequately ablate tissue if they are bouncing or sliding on the tissue surface, in poor contact due 
to low forces, or creating tissue perforations due to large contact forces (Shah et al. 2011, 277; Shah et al. 2010, 1038-1043). The objective of this evaluation was to demonstrate that the robotic catheter system can maintain good target tissue contact and accurately control the applied force in a simulated ablation task. Other representative evaluation tasks that could have also been selected to examine the catheter system include placement of cardiac implants and manipulation of cardiac valve structures (Kesner and Howe 2011c, 1-11; Yuen et al. 2008).

\subsection{Evaluation Method}

The system was evaluated by commanding the catheter to maintain a constant contact force against a moving target. The target was composed of a conductive pad used as the current return path electrode in clinical ablation and electrocautery procedures (REM Polyhesive II Patient Return Electrode, Tyco Healthcare, Gosport, UK) backed with compliant foam (thickness: $25 \mathrm{~mm}$, approximate stiffness: $0.1 \mathrm{~N} / \mathrm{mm}$ ). The target was translated along a $12 \mathrm{~mm}$ amplitude at a frequency of approximately $1 \mathrm{~Hz}$ (60 beats per minute). Two motion patterns were tested: a sinusoidal trajectory and a human mitral annulus trajectory (Kettler et al. 2007, 1290-1295). The ablation quality was approximated by measuring the electrical resistance between the catheter tip electrode and the return electrode pad using an instrumented voltage divider designed to match the approximate resistance range of the catheter tip-target interaction (Figure 17). For the purposes of these validation experiments, resistance also provides an independent measure of contact quality that does not rely on ultrasound imaging. No ablation energy was applied during the electrical resistance measurements. The water tank environment was used to allow the 3DUS guidance system to visualize the catheter and target.

The evaluation experiment was conducted using both the robotic catheter and a commercial manual ablation catheter (RF Marinr MCXL, Medtronic, Minneapolis, Minnesota, USA) for comparison. A manual catheter was select as a passive mechanical equivalent to act as a baseline for comparison in order to analyze the performance of the robotic catheter. For the manual catheter, a load cell was also added to the target to record the forces applied by the catheter tip (LCFD-1KG, Omega Engineering, Stamford, CT, USA; range: 10 N, accuracy: +/$0.015 \mathrm{~N})$. The robotic catheter was instrumented with the force-sensing ablation end effector and was operated under force control with 3DUS guidance. Both catheters were rigidly braced $100 \mathrm{~mm}$ from the ablation tip at orientations perpendicular to the plane of the moving target. 
The manual catheter was positioned so its ablation electrode was able to remain in contact during the entire target trajectory (Figure 18). It should be noted that this experimental setup does not accurately simulate all aspects of intracardiac ablation, including the compliance of the vessels in the heart, the instrument orientation relative to the moving tissue structures, and the electrical impedances of the cardiac environments and human body.

To measure the resistance, $5 \mathrm{~V}$ DC was applied to the patient return electrode and a voltage divider was created at the proximal end of the catheters to measure the ablation resistance (Figure 17). As the contact between the catheter and the target changed due to variations of the contact force or tip position, the resistance between the catheter electrode and the return electrode pad also changed. This resistance, $R_{a b l}$, can be calculated from the resulting output of the voltage divider, $V_{\text {out }}$ :

$$
R_{a b l}=\frac{5 \mathrm{~V}}{V_{\text {out }}} \times 10 \mathrm{k} \Omega-10 \mathrm{k} \Omega=\left(\frac{5 \mathrm{~V}}{V_{\text {out }}}-1\right) \times 10 \mathrm{k} \Omega
$$

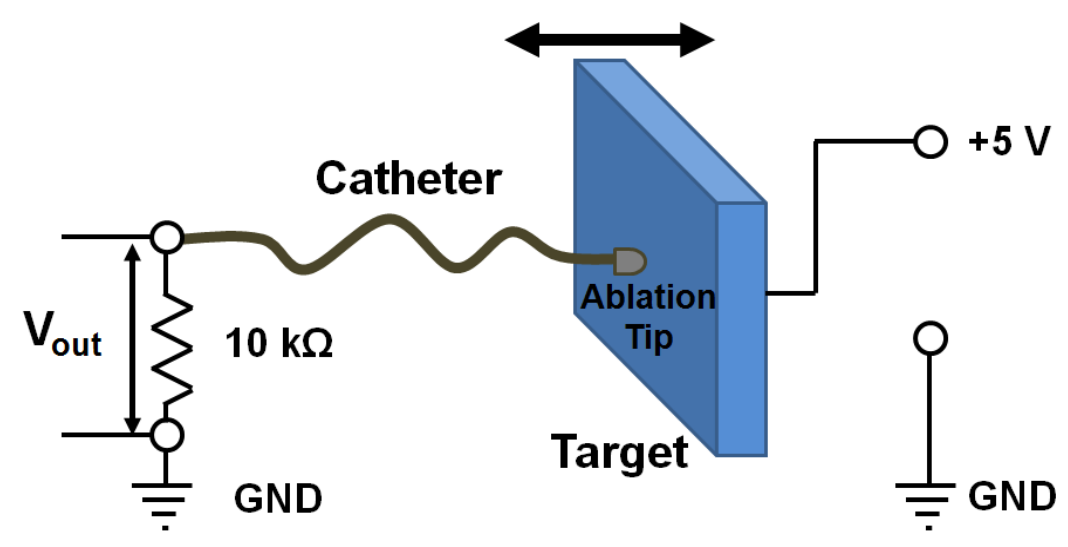

Figure 17: The catheter ablation experimental setup. The moving target was connected to a $5 \mathrm{~V}$ DC signal and the catheters were instrumented with a voltage divider to measure the contact resistance. Resistance measurements were used to evaluate tip contact quality for both a manual catheter and the robotic catheter system. 

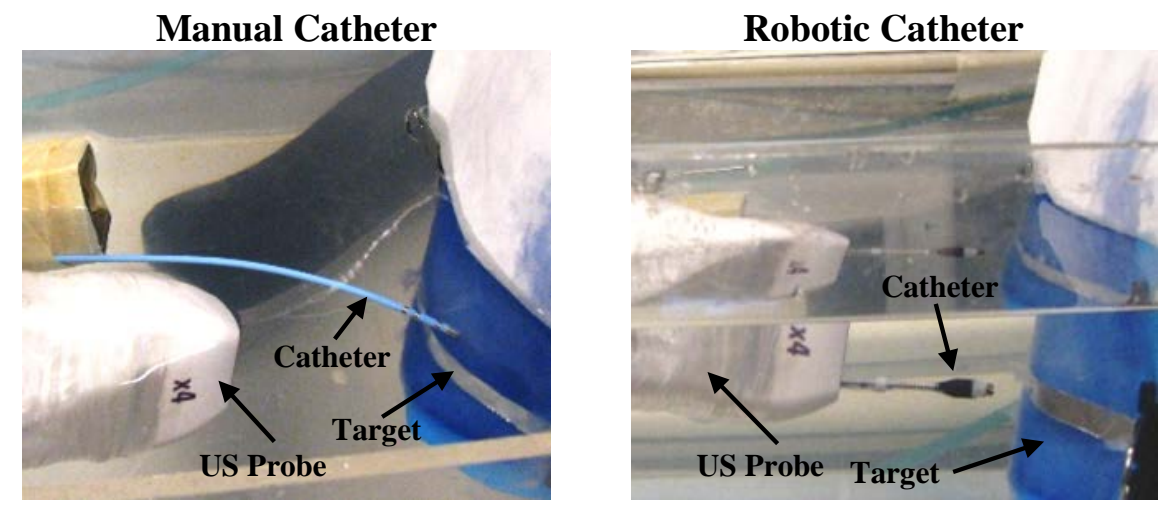

Figure 18: The water tank setup for the manual catheter (top) and the robotic catheter (bottom). Both images show the catheters, the white 3DUS imaging probe, and the motion target.

\subsection{Results}

Figure 19 presents the position trajectories of the 3DUS tracking system, robotic catheter, and motion simulator during the experiments. Figure 20 presents typical results of the ablation experiment on the sinusoidal motion target. Both the manual and robotic catheters were in contact with the moving target for over 5 s during each trial.

The manual catheter was not able to apply a constant force or maintain a constant resistance. The reason for the manual catheter's poor performance was because the motion of the target caused the manual catheter ablation tip to slide and tilt relative to the target surface as the motion simulator pushed on the catheter and caused it to buckle. Compliance is a desirable feature in manual catheters because it prevents them from applying large forces and perforating cardiac tissue. In addition, compliance also allows the catheter to remain in contact despite large motion of cardiac structures. However, this bending compliance makes it challenging to achieve reliable ablation performance. As shown in Figure 20, the manual catheter generated peak-topeak resistance variations of over $20 \mathrm{kOhm}$ and peak-to-peak force variations of over $0.45 \mathrm{~N}$, approximately $125 \%$ of the mean force value $(0.37 \mathrm{~N})$.

The robotic catheter, in contrast to the manual catheter, achieved almost constant resistance values while maintaining a desired force of $1 \mathrm{~N}$ with a force tracking error of 0.11 N RMS. The RMS variation of the resistance value for the robotic catheter was 0.25 kOhms, 97\% less than the RMS variation of $9.88 \mathrm{kOhm}$ for the manual catheter system. The robotic catheter was able to achieve this level of performance because the 3DUS-guided motion 
compensation system and the force control algorithm enabled the ablation tip to maintain consistent contact with the target despite the fast motion (Figure 20).
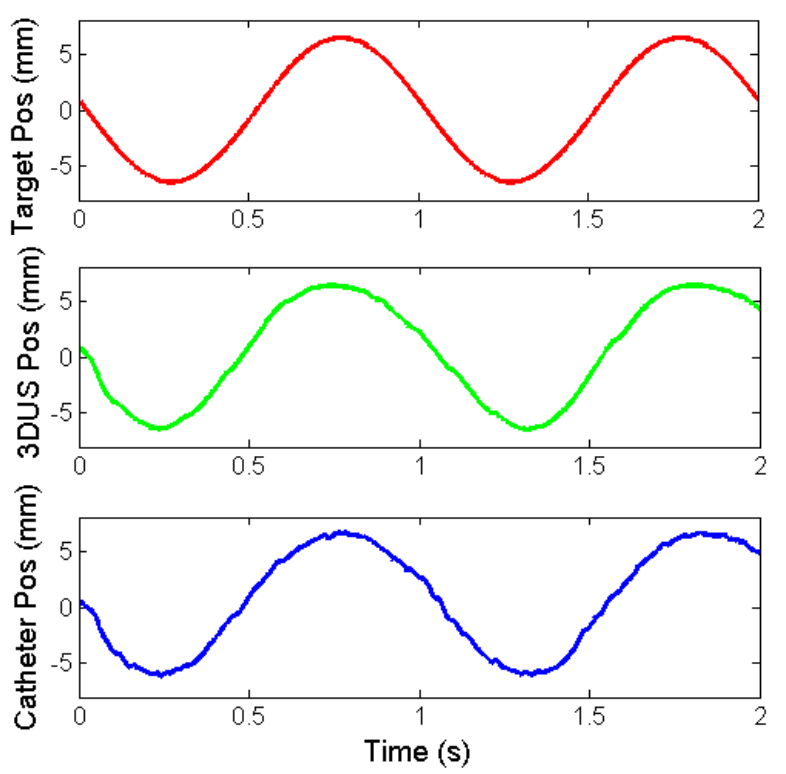

Figure 19: The position trajectories during the robotic catheter experiment: (top) The real target trajectory; (middle) The trajectory determined by the 3DUS tracking system; (bottom) The force-modified position commanded by the control system.

Manual
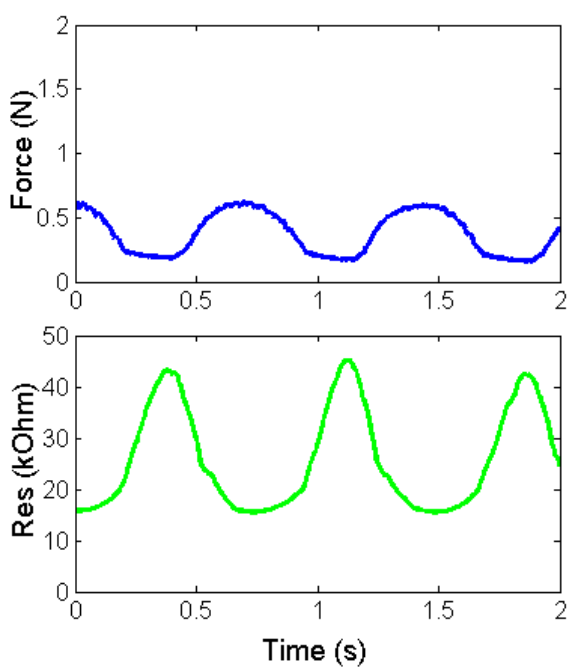

Robotic
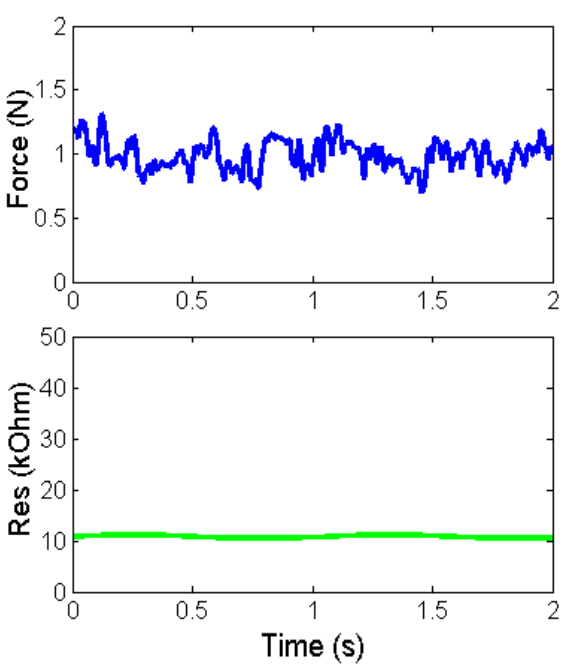

Figure 20: A comparison of the contact resistance and interaction forces between a conductive target and a manual catheter (left) and the robotic catheter system (right). The manual catheter applies a force and resistance that vary with the motion of the target. In contrast, the robotic catheter achieved consistent resistance with the moving target while applying a constant force. 
These results demonstrate that image-guided motion compensation and force control can enable a catheter to maintain a constant, safe force against a moving target, a potentially key feature for improving ablation quality. The system was able to apply a constant force while maintaining a near-constant contact resistance with the ablation end effector on a moving target. In contrast, the force and electrical contact provided by the passive manual catheter in the same experimental setup varied greatly. This variation can primarily be attributed to the buckling, sliding, and tilting behavior of the manual catheter tip due to the target motion. The 3DUS motion tracking enabled the robotic catheter to compensate for the target motion and maintain good ablation electrode contact without the buckling behavior of the manual catheter. It should be noted that a skill clinician operated the manual catheter would have achieved better results than the passive mechanical equivalent through improved catheter tip position manipulation and actively adjusting the angle of the catheter relative to the moving target surface. The required position and force accuracy for the robotic catheter system depends on the specific clinical application, the required quality of interventional procedure, and the force levels that will result in tissue damage for the application. An argument can be made that an accuracy roughly similar to a skilled surgeon operating on a stopped heart with an actuated instrument would be appropriate for this work. Thus, a position accuracy of approximately $1 \mathrm{~mm}$ was deemed sufficient (Falk 2002, 624-628). Force accuracy is a more challenging value to select. A force accuracy equivalent or better than a human hand performing a dexterous manipulation task is appropriate. Jones and Piateski found that for finger and hand grasping tasks, an average force accuracy of roughly $0.5 \mathrm{~N}$ was attainable for a 2-10 range (Jones and Piateski 2006, 298-302). Our system will apply smaller forces than this range, thus an accuracy of under $0.25 \mathrm{~N}$ is sufficient.

One insight from this work is that multiple forms of sensor information are required to command a catheter to safely and effectively interact with the moving target. Force sensing alone is not sufficient for the catheter to track the target motion, as described in (Kesner and Howe 2011b, 1589-1594). This is due to the fact that the catheter performance limitations of backlash and friction prevent the system from responding fast enough to the quick tissue motion using only force feedback. Motion tracking must also be used to overcome these limitations and maintain system stability (Kesner and Howe 2011b, 1589-1594). The image guidance provides an 
approximation of the desired position trajectory for the tip of the catheter and the force feedback introduces minor adjustments to the tip position to regulate and maintain the tool-tissue interactions forces. This insight is demonstrated with the $20 \%$ position tracking improvements provided by the combination of the force control system and 3DUS motion tracking while following the mitral valve annulus motion simulator, as shown in Figure 16Error! Reference source not found.. Without either 3DUS guidance or force sensing, the catheter would be unable to maintain the consistent ablation electrode contact and could either penetrate or retract from the target surface.

In addition to 3DUS, other position tracking systems are available for intracardiac interventions. For example, ablation impedance tracking systems, such as the CARTO system (Biosense Webster, Inc., Diamond Bar, CA, USA), use a combination of the ablation catheter position from electromagnetic tracking and the electrical impedance values to perform cardiac mapping and ablation procedures. While these systems do assist clinicians to guide ablation procedures, they do not track the physical position of the moving cardiac tissue in real time to allow a catheter to compensate for the motion or apply a constant force against moving cardiac structures. 3DUS enables the advantages of imaging and tracking both the catheter and soft tissue to allow for safe manipulation of fast-moving cardiac tissues.

Although the experimental results demonstrate that the robotic catheter system is able to apply a constant force while maintaining a consistent ablation contact, there are a number of limitations in this initial validation study due to the challenges of accurately simulating in vivo cardiac ablation in a laboratory setting. First, measuring the DC resistance of the contact does not consider the electrical frequency response of the cardiac tissue at the $500 \mathrm{kHz}$ frequency used by the RF energy generator. In addition, the system was tested in water instead of electricallyconductive blood or saline, which alters the electrode conduction properties. Finally, the experimental setup did not approximate the exact mechanics of intracardiac ablation, including the compliance of the vessels in the heart and the tool orientation relative to the moving tissue structures. The manual catheter performance depends on its orientation with respect to the moving tissue target, although similar fluctuations in force and resistance would have resulted for other orientations. We anticipate that these issues will not impair the demonstrated advantages of the robotic system because of the known properties of the ablation process and the 
success of previous in vivo tests of the image guidance systems (Kesner and Howe 2011c, 1-11; Yuen et al. 2008; Kesner, Yuen, and Howe 2010, 168-178).

\section{$7 \quad$ Conclusions}

This paper presents the experimental evaluation of a robotic catheter system for interacting with the beating heart. The specific medical application selected for this system is performing tissue ablation on a moving cardiac structure. The system uses motion compensation and force feedback to maintain a constant force and contact resistance on a moving target. The experimental results presented here demonstrated that the robotic system is able to apply a constant force on a moving target with $0.08 \mathrm{~N}$ RMS error. In addition to accurately controlling the catheter-tissue interaction forces, the system is able to maintain consistent ablation electrode contact against a translating motion simulator with a 97\% reduction in RMS resistance variation over a passive and rigidly fixed manual catheter. This ablation result can be explained by the fact that a compliant manual catheter slides and buckles while in contact with a quickly moving structure, such as the actively contracting heart wall, while the robotic catheter actively compensates for the motion and regulates the interaction forces. Finally, the combination of force feedback and 3DUS guidance improves the trajectory tracking accuracy by adjusting the catheter motion due to the forces experienced at the catheter tip while interacting with the moving target.

Future work in this project will focus on the demonstration and evaluation of the technology in an in vivo setting. While the motion compensation and robotic catheter system has been demonstrated previously in vivo (Yuen et al. 2008; Kesner, Yuen, and Howe 2010, 168-178; Kesner and Howe 2011c, 1-11), the force control ablation system has not yet been tested inside a beating heart. One possible challenge the system will encounter in vivo is how to respond as the tissue stiffness changes over the course of the heart cycle. In addition, safety issues such as system stability and preventing tissue collisions will need to be further investigated. The project objective is to enable a range of beating heart surgical procedures with a catheter, and the ablation application explored here is a first step toward this ultimate goal. 


\section{References}

Baim, Donald S., ed. 2005. Grossman's Cardiac Catheterization, Angiography, and Intervention. Philadelphia, PA: Lippincott Williams \& Wilkins.

Bebek, O. and M. Cavusoglu. 2007. "Intelligent Control Algorithms for Robotic Assisted Beating Heart Surgery." IEEE Trans. on Robotics 23 (3): 468-480.

Beyar, R. 2010. "Navigation within the Heart and Vessels in Clinical Practice." Annals of the New York Academy of Sciences 1188 (1): 207-213.

Camarillo, D. B., C. F. Milne, C. R. Carlson, M. R. Zinn, and J. K. Salisbury. 2008. "Mechanics Modeling of Tendon-Driven Continuum Manipulators." Robotics, IEEE Transactions On 24 (6): 1262-1273.

Chiaverini, S., B. Siciliano, and L. Villani. 1999. "A Survey of Robot Interaction Control Schemes with Experimental Comparison." Mechatronics, IEEE/ASME Transactions On 4 (3): 273-285.

Eppinger, S. and W. Seering. 1987. "Understanding Bandwidth Limitations in Robot Force Control."IEEE, .

Falk, Volkmar. 2002. "Manual Control and Tracking-a Human Factor Analysis Relevant for Beating Heart Surgery." The Annals of Thoracic Surgery 74 (2): 624-628.

Ginhoux, R., J. Gangloff, M. de Mathelin, L. Soler, M. M. A. Sanchez, and J. Marescaux. 2005. "Active Filtering of Physiological Motion in Robotized Surgery using Predictive Control." Robotics, IEEE Transactions On 21 (1): 67-79.

Huang, S. K., S. K. S. Huang, and D. J. Wilber. 2000. Radiofrequency Catheter Ablation of Cardiac Arrhythmias: Basic Concepts and Clinical Applications Wiley-Blackwell.

Jayender, J., M. Azizian, and R. V. Patel. 2008. "Autonomous Image-Guided Robot-Assisted Active Catheter Insertion." Robotics, IEEE Transactions On 24 (4): 858-871.

Jones, Lynette A. and Erin Piateski. 2006. "Contribution of Tactile Feedback from the Hand to the Perception of Force." Experimental Brain Research 168 (1): 298-302.

Kalman, J. M., A. P. Fitzpatrick, J. E. Olgin, M. C. Chin, R. J. Lee, M. M. Scheinman, and M. D. Lesh. 1997. "Biophysical Characteristics of Radiofrequency Lesion Formation in Vivo: Dynamics of Catheter Tip-Tissue Contact Evaluated by Intracardiac Echocardiography." American Heart Journal 133 (1): 8-18.

Kesner, S. B., S. Yuen, and R. Howe. 2010. "Ultrasound Servoing of Catheters for Beating Heart Valve Repair." Information Processing in Computer-Assisted Interventions: 168-178.

Kesner, S. B. and R. D. Howe. 2010. "Design and Control of Motion Compensation Cardiac Catheters."IEEE, . 
_ 2011a. "Design Principles for Rapid Prototyping Forces Sensors using 3-D Printing."

Mechatronics, IEEE/ASME Transactions On (99): 1-5.

— 2011b. "Force Control of Flexible Catheter Robots for Beating Heart Surgery."IEEE, .

_ 2012. "Motion Compensated Catheter Ablation of the Beating Heart using Image Guidance and Force Control." Quebec City, Canada, .

_ 2011c. "Position Control of Motion Compensation Cardiac Catheters." Robotics, IEEE Transactions On (99): 1-11.

Kettler, D. T., R. D. Plowes, P. M. Novotny, N. V. Vasilyev, P. J. del Nido, and R. D. Howe. 2007. "An Active Motion Compensation Instrument for Beating Heart Mitral Valve Surgery.".

Maples, J. and J. Becker. 1986. "Experiments in Force Control of Robotic Manipulators."IEEE, .

Murkin, J. M., W. D. Boyd, S. Ganapathy, S. J. Adams, and R. C. Peterson. 1999. "Beating Heart Surgery: Why Expect Less Central Nervous System Morbidity?" The Annals of Thoracic Surgery 68 (4): 1498-1501.

Nakamura, Y., K. Kishi, and H. Kawakami. 2001. "Heartbeat Synchronization for Robotic Cardiac Surgery.".

Novotny, P. M., J. A. Stoll, N. V. Vasilyev, P. J. Del Nido, P. E. Dupont, T. E. Zickler, and R. D. Howe. 2007a. "GPU Based Real-Time Instrument Tracking with Three-Dimensional Ultrasound." Medical Image Analysis 11 (5): 458-464.

Novotny, P. M., J. A. Stoll, P. E. Dupont, and R. D. Howe. 2007b. "Real-Time Visual Servoing of a Robot using Three-Dimensional Ultrasound.".

Objet Geometries Ltd. "FullCure870 VeroBlack Data Sheet." Objet Geometries Ltd2010.

Okumura, Y., S. Johnson, and D. Packer. 2007. "An Analysis of Catheter Tip/Tissue Contact ForceInduced Distortion of Three-Dimensional Electroanatomical Mapping Created using the Sensei Robotic Catheter System." Heart Rhythm 4: S318.

Prisco, G. M. Fiber Optic Shape Sensing.

Richa, R., A. P. L. Bó, and P. Poignet. 2010. "Beating Heart Motion Prediction for Robust Visual Tracking."IEEE, .

Roach, G. W., M. Kanchuger, C. M. Mangano, M. Newman, N. Nussmeier, R. Wolman, A. Aggarwal, K. Marschall, S. H. Graham, and C. Ley. 1996. "Adverse Cerebral Outcomes After Coronary Bypass Surgery." New England Journal of Medicine 335 (25): 1857-1864.

Shah, D., H. Lambert, A. Langenkamp, Y. Vanenkov, G. Leo, P. Gentil-Baron, and B. Walpoth. 2011. "Catheter Tip Force Required for Mechanical Perforation of Porcine Cardiac Chambers." Europace 13 (2): 277. 
Shah, D. C., H. Lambert, H. Nakagawa, A. Langenkamp, N. Aeby, and G. Leo. 2010. "Area Under the Real-Time Contact Force Curve (Force-Time Integral) Predicts Radiofrequency Lesion Size in an in Vitro Contractile Model." Journal of Cardiovascular Electrophysiology 21 (9): 1038-1043.

Townsend, W. and J. Salisbury Jr. 1987. "The Effect of Coulomb Friction and Stiction on Force Control."IEEE, .

Wagner, Christopher R., Douglas P. Perrin, Robert D. Howe, Nikolay Vasilyev, and Pedro J. Del Nido. 2006. "Force Feedback in a Three-Dimensional Ultrasound-Guided Surgical Task."IEEE, .

Webster, J. G. 1988. Tactile Sensors for Robotics and and Medicine. New York: John Wiley \& Sons, Inc.

Wilson, E., Z. Yaniv, D. Lindisch, and K. Cleary. 2008. "A Buyer’s Guide to Electromagnetic Tracking Systems for Clinical Applications.".

Yuen, S. G., D. T. Kettler, P. M. Novotny, R. D. Plowes, and R. D. Howe. 2009. "Robotic Motion Compensation for Beating Heart Intracardiac Surgery." The International Journal of Robotics Research 28 (10): 1355.

_ 2010. "Robotic Tissue Tracking for Beating Heart Mitral Valve Surgery." Medical Image Analysis.

Yuen, S. G., S. B. Kesner, Nikolay V. Vasilyev, P. J. del Nido, and R. D. Howe. 2008. "3D UltrasoundGuided Motion Compensation System for Beating Heart Mitral Valve Repair.".

2008. "Quasiperiodic Predictive Filtering for Robot-Assisted Beating Heart Surgery.". 